Portland State University

PDXScholar

1990

\title{
Goal-directed imagining : the effect of suggestions of warmth and coolness on blood flow to the hand
}

John Mark Dilworth

Portland State University

Follow this and additional works at: https://pdxscholar.library.pdx.edu/open_access_etds

Part of the Psychology Commons

Let us know how access to this document benefits you.

\section{Recommended Citation}

Dilworth, John Mark, "Goal-directed imagining : the effect of suggestions of warmth and coolness on blood flow to the hand" (1990). Dissertations and Theses. Paper 3981.

https://doi.org/10.15760/etd.5862

This Thesis is brought to you for free and open access. It has been accepted for inclusion in Dissertations and Theses by an authorized administrator of PDXScholar. Please contact us if we can make this document more accessible: pdxscholar@pdx.edu. 
AN ABSTRACT OF THE THESIS OF John Mark Dilworth for the

Master of Science in Psychology presented July 16, 1990.

Title: Goal-directed Imagining: The Effect of Suggestions of Warmth and Coolness on Blood Flow to the Hand.

APPROVED BY THE MEMBERS OF THE THESIS COMMITTEE:
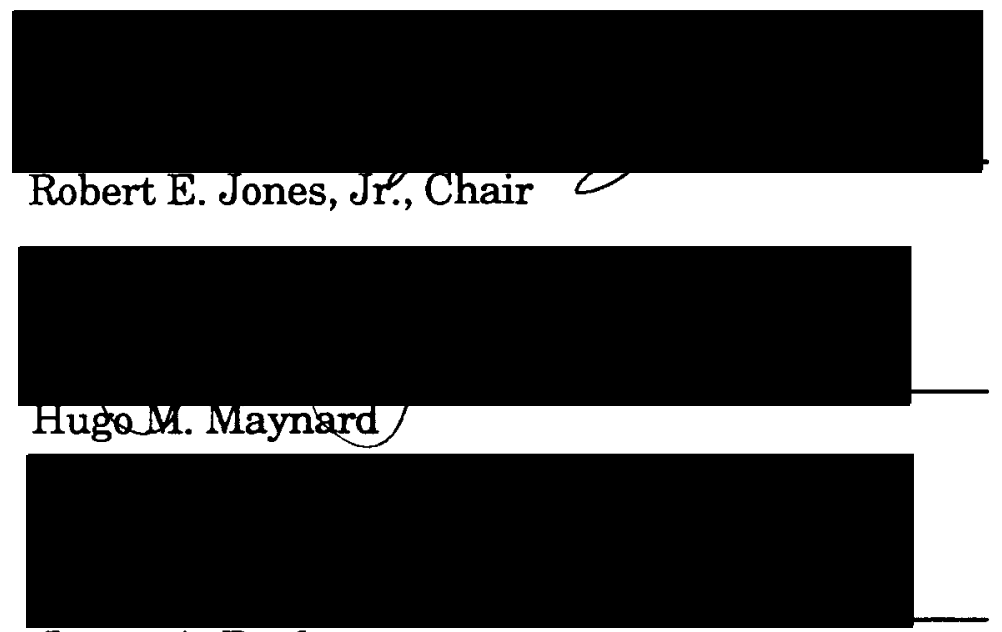

$$
\text { James A. Paulson }
$$

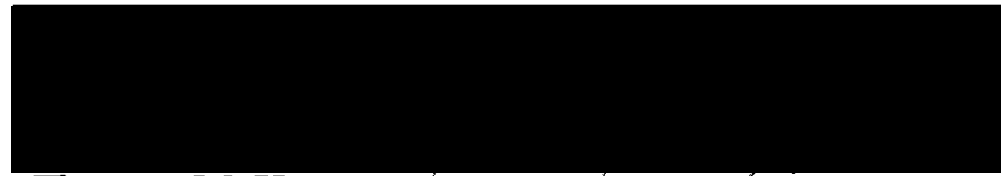

Thomas M. Young

Recent research was reviewed which claimed to demonstrate that hypnotic suggestions could be used to control blood flow. Numerous methodological and conceptual problems in these studies were identified and a rigid experimental design with tighter controls was employed to investigate the claimed effects on blood flow. Subjects listened to either a 
standardized hypnotic induction or a passage of relaxing music. Both groups then listened to the Creative Imagination Scale (Wilson \& Barber, 1978) (CIS) which ended with an added item containing suggestions of coolness. Localized skin temperature of the right hand was monitored throughout as an indication of blood flow.

No significant blood flow increases in response to suggestions of warmth nor decreases in response to suggestions of coolness were observed. The experience of suggested events did not differ significantly between those subjects who received the induction and those who received the passage of music.

An increase in blood flow occurred in response to receiving either an induction or music. There was, however, no significant difference between these two groups on the magnitude of the increase. Neither was there a significant difference in this magnitude between high scorers on the CIS and low scorers on the CIS. Results of this and previous studies were discussed within the context of the effects of relaxation as an alternative explanation to the supposed effects of hypnotic suggestion. 
GOAL-DIRECTED IMAGINING: THE EFFECT OF SUGGESTIONS OF WARMTH AND COOLNESS ON BLOOD FLOW TO THE HAND

\author{
by \\ JOHN MARK DILWORTH
}

A thesis submitted in partial fulfillment of the

requirements for the degree of

MASTER of SCIENCE

in

PSYCHOLOGY

Portland State University

1990 
TO THE OFFICE OF GRADUATE STUDIES:

The members of the Committee approve the thesis of John Mark Dilworth presented July 16, 1990.

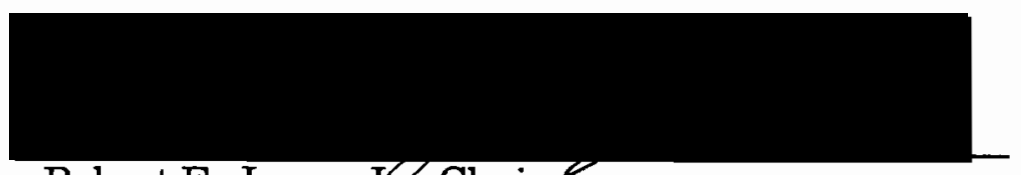

Robert E. Jones, Jre., Chair

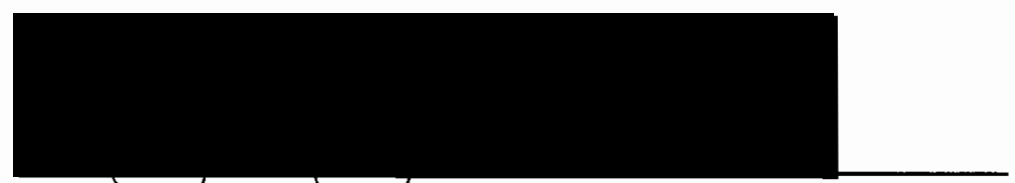

HugdM.Maynard

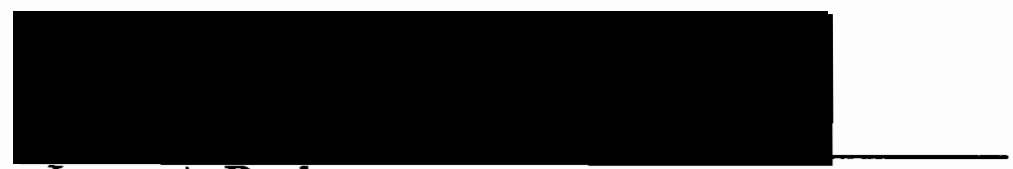

James A.Paulson

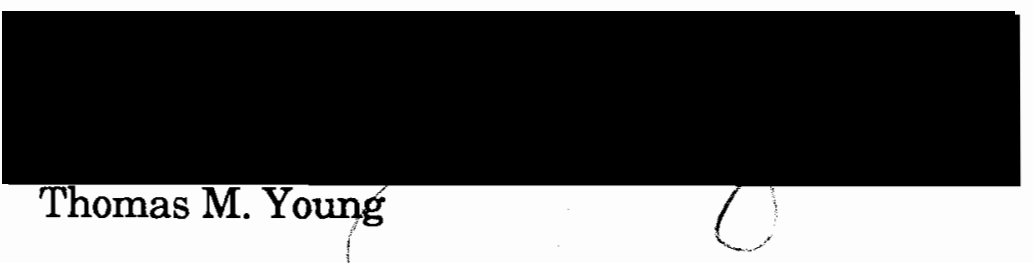

APPROVED:

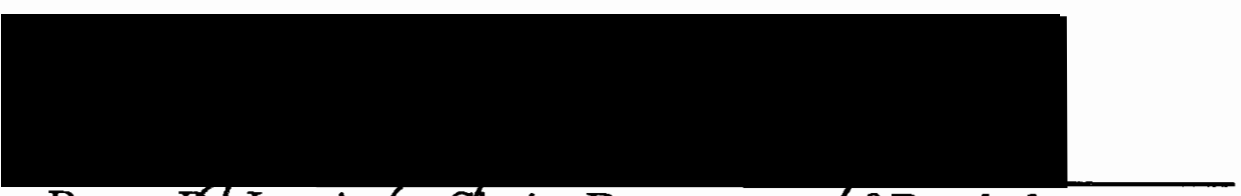

Roger $\not \delta / J e n n i n \$ s$, Ghair, Department/of Psychology

C. William Savery, Interim Vice Provost for Graduate

Studies and Research 


\section{TABLE OF CONTENTS}

LIST OF TABLES $\ldots \ldots \ldots \ldots \ldots \ldots \ldots \ldots \ldots \ldots \ldots \ldots \ldots$

LIST OF FIGURES $\ldots \ldots \ldots \ldots \ldots \ldots \ldots \ldots \ldots \ldots \ldots$

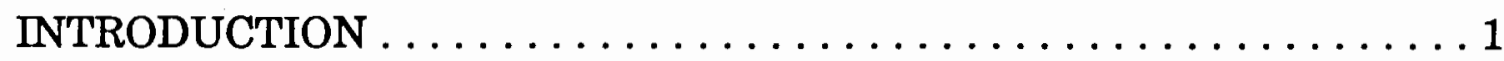

Effects of Suggestion on Blood Flow: Recent Research . . . . . . . 2

Response to Suggestion as Goal-directed Imagining . . . . . . . 7

The Measurement of Goal-directed Imagining $\ldots \ldots \ldots \ldots$

Focus of the Study $\ldots \ldots \ldots \ldots \ldots \ldots \ldots \ldots \ldots$

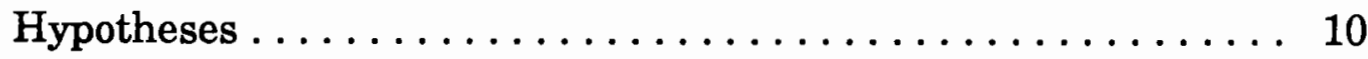

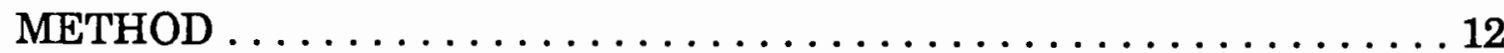

Subjects $\ldots \ldots \ldots \ldots \ldots \ldots \ldots \ldots \ldots \ldots \ldots \ldots \ldots \ldots \ldots \ldots \ldots$

Measures ........................ 12

Conditions ........................ 13

Procedure $\ldots \ldots \ldots \ldots \ldots \ldots \ldots \ldots \ldots \ldots \ldots \ldots \ldots \ldots$

RESULTS . . . . . . . . . . . . . . . . . 16

Test of Hypotheses . . . . . . . . . . . . . . . . 19

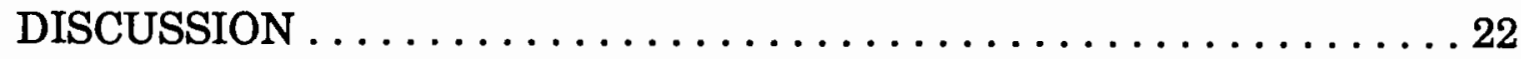

REFERENCES . . . . . . . . . . 26 


\section{APPENDICES}

A SUMMARY OF CIS ITEMS $\ldots \ldots \ldots \ldots \ldots \ldots \ldots \ldots$

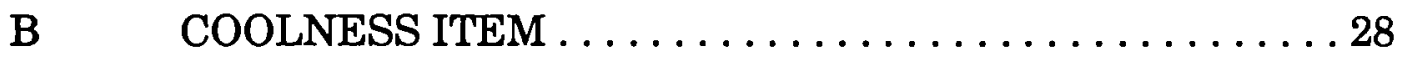

C SCREENING INSTRUMENT . . . . . . . . . . 29 


\section{LIST OF TABLES}

I $\quad$ Mean Skin Temperature by Treatment $\ldots \ldots \ldots \ldots \ldots \ldots \ldots$

II Group Sizes: Condition and CIS $\ldots \ldots \ldots \ldots \ldots \ldots \ldots$

III Mean Skin Temperature by Group for Induction/Music,

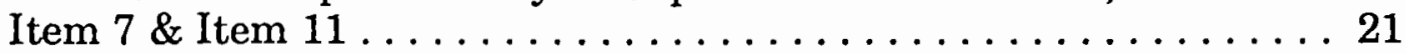




\section{LIST OF FIGURES}

1. Mean skin temperature as a function of treatment time $\ldots \ldots \ldots 18$ 


\section{INTRODUCTION}

Recent investigations into the effect of hypnotic suggestion on changes in blood flow have put forth claims that hypnosis is a valuable resource in the treatment of burn wounds (Hammond et al., 1983; Moore \& Kaplan, 1983; Wakeman \& Kaplan, 1978), warts (Clawson \& Swade, 1975), Raynaud's disease (Grabowska, 1971) and hemophilia (Swirsky-Sacchetti \& Margolis, 1986). Other investigations have reported positive relationships between suggestion and changes in blood flow (Bishay \& Lee, 1984; Erickson, 1980). Although, on the face of things, the findings of these studies seem impressive, the studies themselves were not without an array of methodological and measurement shortcomings which call into question the validity of their claims. Many of these studies failed to statistically test the differences which were reported. Many failed to control for crucial factors which may have influenced the reported outcomes. Many failed to take into account the introduction of possible confounds as a result of lengthy treatment intervals. Finally, most of the studies lacked a standardized measure of hypnotic susceptibility.

It could be that these methodological shortcomings were, in part, the result of the lack of a strong theoretical position on hypnotic phenomena which, ideally, should have driven the researchers in their choice of design by providing a firm foundation from which to proceed. Sheehan \& Perry (1980), in addressing the role of theory for experimental hypnosis designs, 
suggested that:

Where method is under scrutiny, there must be adequate recognition of the role theory has to play in deciding which procedures are suitable for use (p. 517)

and

The anomaly of research in hypnosis is that it is operationally difficult to index what exactly it is about experimental treatments (such as standard induction instructions) which actually constitutes hypnosis ... hypnotic induction has a multitude of characteristics which define it and many of them give rise to comparable effects occurring in contexts that are clearly not defined as hypnosis. It is important to decide on a criterion for the presence or absence of hypnosis. (p. 533)

Thus, a crucial component of a design is a measure of the extent to which subjects experience the phenomena purported to account for any observed differences. Use of a measure of hypnotic susceptibility affords the opportunity for inferences based on differences in trance depth (i.e., statebased hypotheses) or differences in suggestion-related abilities (i.e., traitbased hypotheses). Selection of a specific measure should be guided by a particular paradigm which the researcher either adheres to or seeks to test.

\section{EFFECTS OF SUGGESTION ON BLOOD FLOW: RECENT RESEARCH}

Grabowska (1971) reported that suggestions of warmth to a subject's hand resulted in mean increases of 2-7 degrees Celsius in the target hand. Using state-based assumptions regarding the observed phenomena, two "stages" of hypnotic depth were reported. No information was provided concerning how trance depth was measured. The second, purportedly "deeper", stage was characterized by the previously mentioned increases in skin temperature. Over a period of four weeks, 15,000 measurements were 
averaged across 420 suggestions to arrive at the reported suggestion-related increases in blood flow.

Unfortunately, a number of non-hypnotic interventions (e.g., relaxation training) were incorporated into the design. Failure to control for these, in addition to an indeterminability of the homogeneity of suggestions, suggests an alternative explanation in which variations in the activity a subject is engaged in may account for blood flow increases independent of any state-specific hypnotic phenomena. It is interesting to note that a "spontaneous increase" (p. 1044) in skin temperature occurred prior to suggestion of warmth. It could be that a trait-based anticipation factor was responsible for the purportedly state-dependent increases.

Clawson \& Swade (1975) reported four case studies in which suggestions to decrease blood flow were used to reduce bleeding and to eliminate warts. Depth of hypnosis was determined by "passing under his nose a small bottle containing concentrated ammonia. There was no evidence that the caustic aroma ... had any effect on him" (p. 161). A single authoritative suggestion to "stop this bleeding" (p. 161) resulted in decreased blood flow in two of the cases. In the other two cases, multiple wart patients were given suggestions to "stop the blood supply to each wart on your body" (p. 163) over a period of four weeks. During the period, the number of warts decreased progressively until all warts were gone. The authors admitted, with respect to the disappearance of warts, that "spontaneous remission is often encountered" (p. 164).

Erickson (1980) presented a brief report in which alternate suggestions 
of warmth and coldness resulted in increases and decreases, respectively, of blood flow. A waking state control group was given the suggestions with no changes in blood volume (as measured by a plethysmograph). Although "significant recordings" (p. 194) were claimed for the hypnotic state group, no reference to the specific statistical tests used to assess the relationship between suggestion and blood volume was given. Also, no criterion for distinguishing between the waking state group and the hypnotic state group was reported. The waking state group was described as:

The subjects were instructed simply: "For five minutes, in silence, you are to watch that clock and, while doing so, you are to make your right hand cold and your left hand hot. Then for another five minutes, you will reverse the procedure by making the right hand hot and the left hand cold." (p. 193)

and the hypnotic state group was described as:

... merely a repetition of the first procedure. This time, however, the subject was in a profound somnambulistic trance with instructions to be able to hear and understand the colleague. (p. 193)

Hammond et al. (1983) investigated the effect of suggestions of coolness on inflammation and the healing of first degree burns. A subjectas-own-control design was employed in which one leg received a first degree burn. Assignment to condition was made on the basis of a coin toss. Thus, some subjects received suggestions of coolness for the burned leg (experimental condition) while others received the suggestions for the nonburn leg (control condition).

Ratings of redness were significantly less for those subjects whose burned legs were in the experimental condition (compared to those whose 
burned legs were in the control condition). The experimental group showed significantly lower burn area skin temperatures than the control burned leg group. The study also investigated the relationship between hypnotic susceptibility (as measured by the Stanford Hypnotic Clinical Scale) and dependent variables. No significant relationship between susceptibility and either skin temperature or degree of redness was observed although the exact magnitudes of the correlations tested were not reported.

Moore \& Kaplan (1983) examined the effect of suggestion on the acceleration of second degree burn wound healing. Bilaterally burned subjects (e.g., burns on both legs in equidistant locations and covering similar regions) received suggestions to one side of their burned body areas. The authors, using a case study format, claimed that the treatment side healed, on the average, three days faster than the control side. However, no statistical tests of whether this faster rate was significant were reported. No information about the kind of suggestions used was included. No attempt to examine the relationship between susceptibility and healing rate was made.

Bishay \& Lee (1984) reported a positive relationship between suggestions to subjects of imagery in which one hand was immersed in cold water and subsequent reduction of blood flow in the target hand. Using hand levitation performance as a measure of "depth of hypnotic trance" (p. 66), the authors reported that most of the subjects exhibited light or moderate levels of depth. In agreement with Grabowska (1971), 
increases in blood flow were observed prior to the suggestions.

Swirsky-Sacchetti \& Margolis (1986) reported a study in which hemophiliacs who participated in a six-week training program "significantly reduced the amount of factor concentrate used to control bleeding in comparison to controls" (p. 71). Although the authors claimed that hypnosis was responsible for the observed changes, a treatment effect can't be attributed directly to hypnosis because other training variables (education, support, relaxation) may have interacted with or mediated hypnosis effects. These variables were not controlled for, a shortcoming that the authors noted. A moderate negative correlation between improvement and suggestibility (as measured by the Harvard Group Scale of Hypnotic Susceptibility) suggested that other training factors may have been responsible for the reported improvement.

In a well-controlled study that failed to yield effects of suggestion on changes in skin temperature, Spanos et al. (1982) age-regressed subjects who had been burned previously (at least six months prior to the study). Repeated imagery suggestions to "relive the original burn experience" (p. 304) and that "a blister was forming" (p. 303) did not result in expected increases in skin temperature for 59 of 60 subjects. Susceptibility was measured by the Harvard Group Scale of Hypnotic Susceptibility: Form A and failed to yield a significant relationship with skin temperature changes. Of interest was the observation that, of the total sample, the sole subject to demonstrate an effect between suggestion and temperature showed only medium susceptibility. Subjects responded to a post-experiment 
questionnaire designed to tap experiential aspects of the experimental task. $53 \%$ of the subjects reported that the experienced imagery was at least $75 \%$ as vivid as the original experience (i.e., the actual "burn event" occurring in the past) had been.

In summary, the preceding studies lacked sufficient methodology to support their findings. Although seven of the eight studies reported effects of suggestion on blood flow alteration, only one of these studies attempted to address any theoretical implications of the reported effects. This sole study by Swirsky-Sacchetti \& Margolis (1986) posited that the subjects who demonstrated an effect were "capable of using their knowledge and skills to achieve the noted effects" (p. 79). Thus, while stated rather vaguely, the implication was that "successful subjects" were successful to the extent that trait-based variables were present (i.e., using knowledge and skills).

\section{RESPONSE TO SUGGESTION AS GOAL-DIRECTED IMAGINING}

Barber and De Moor (1972) postulated nine variables as determinants of successful response to hypnotic suggestions. Of particular importance to the present study are the two variables "coupling suggestions with naturally occurring events [and] stimulating goal-directed imagining" (p. 132). Goal-directed imagining was operationalized as "imagining a situation which, if it actually occurred, would tend to give rise to the behavior that was suggested" (Barber et al., 1974, p. 60).

The ability to produce goal-directed imagining appears to be related to successful selective amnesia (Spanos \& Ham, 1973) and successful hand 
levitation (Spanos \& Barber, 1972). In a paper which focused on the traitbased paradigm that encompasses the construct of goal-directed imagining, Spanos and Barber (1974) noted that:

The nonstate view of hypnotic behavior grew ... out of a movement aimed at curtailing the excessive claims made by some state theorists. This viewpoint became associated first with the notion that hypnotic behaviors did not involve highly unusual or transcendent phenomena and, second, with the notion that it was unnecessary and misleading to posit a special or qualitatively different state in order to explain the experiences of the good hypnotic subject. (p. 508)

Thus, the nonstate, trait-based paradigm implies that successful response to hypnotic suggestions is determined, to some extent, by abilities of the subject (e.g., goal-directed imagining) as opposed to some special characteristics of a hypnotic state or trance.

\section{THE MEASUREMENT OF GOAL-DIRECTED IMAGINING}

The Creative Imagination Scale (CIS) (Wilson \& Barber, 1978) grew out of attempts to develop a measure of subjects' goal-directed imaging in response to hypnotic suggestions. According to the authors' instructions, the 10-item scale may be administered with or without an induction. The CIS is actually a revision of the earlier Barber Suggestibility Scale (BSS) (Barber, 1969). The CIS items purportedly differ from the BSS items in that they are more permissive in their phrasing (e.g., "By using your imagination constructively, you can ...") as opposed to the authoritarian phrasing of the BSS items.

Barber and Wilson (1978) explained the purposes for which the BSS and CIS were designed: 
We needed a representative series of standardized test suggestions that a) could be administered relatively quickly, easily, and without special equipment, b) could be used in a situation defined to the subject either as hypnosis or a test of imagination, and c) could be given to subjects who had, and also to those who had not, been exposed to a hypnotic induction procedure. (p. 84)

Information on the validity and reliability of the CIS is sparse. Wilson and Barber (1978) reported test-retest reliability of .82 for a sample of 22 and split-half correlation of .89 for a sample of 217 . The authors also reported a factor analysis which resulted in a one factor solution. The CIS correlated .60 with the BSS. McConkey et al. (1979) reported a correlation of .28 between the CIS and the Harvard Group Scale of Hypnotic Susceptibility: Form A. The authors also reported a one factor solution for the 10 CIS items. De Horne and Powlett (1980), in reviewing the CIS, emphasized that reliability and validity "claims need to be independently substantiated" (p. 127). Appendix A summarizes the ten CIS items.

\section{FOCUS OF THE STUDY}

The present study investigated the extent to which involvement in goal-directed imagining elicits changes in localized skin temperature to a target hand. The seventh item of the CIS, labeled Temperature Hallucination, consists of suggestions of warmth to the right hand. Changes in skin temperature in the right hand were measured throughout administration of the CIS (with particular analytical emphasis on item 7) to assess the relationship between goal-directed imagining and increase in blood flow. A separate analysis of potential 
decreases in blood flow as a response to suggestions of cold and numbness was afforded by the addition of the suggestions of Bishay and Lee (1984) following CIS item 10.

Administration of the CIS permitted groupings of subjects into high, medium and low levels of goal-directed imagining with the norms presented by Wilson and Barber (1978) used as an adjunct in defining the specific cutoff scores for the groups. A control group listened to a passage of relaxing music in place of a formal induction in order to assess the validity of the CIS for non-induction procedures and to determine whether the administration of a standardized induction made any difference a) in potential changes in blood flow or b) in subjects' experiential reports of involvement in goal-directed imagining. It could be that the claimed effect of hypnotic suggestion on increases in blood flow (e.g., Grabowska, 1971) is really an effect of the hypnotic induction. As an induction is, in part, a series of progressive relaxation instructions, it would follow that, as the subject receiving the induction relaxed more and more, blood flow to the extremities increased as a function of relaxation.

Specifically, the assumption was made that hypnotic performance phenomena could be attributed to traits, or abilities, of the subject. Hence, following the lead of Barber (1979), there is no reason to posit a special state that differs from the normal waking state for explaining individual differences in hypnotic performance. If this were indeed the case, hypnotic performance would not be expected to differ as a result of induction.

Specific hypotheses were made concerning state vs. trait paradigms. 
It was hypothesized that changes in blood flow in response to suggestions would vary independent of condition as would subjects' experiences of the suggested events. It was also hypothesized that high scorers on the CIS would perform better than low scorers on the items designed to suggest increases and decreases in blood flow.

Two more exploratory hypotheses were made concerning expected increases in blood flow as a result of induction or listening to relaxing music. It was hypothesized that a rise in blood flow would occur in response to these treatments and that there would be no difference between conditions on that rise. It was also hypothesized that high scorers on the CIS would demonstrate a greater rise in blood flow than low scorers in response to the induction/music treatment. 


\section{METHOD}

\section{SUBJECTS}

36 subjects ( 22 females and 14 males) were recruited, on a volunteer basis, from undergraduate psychology classes at Portland State University in Portland, Oregon. Subjects received extra credit from their class professor for participation. During recruitment, the experiment was described as a study on the imagination and on the extent to which people may be able to use their imagination as a tool in relaxation and health. The mean age of the subjects was 32.33 with a range of 20 to 44 years.

\section{MEASURES}

\section{Goal-directed Imagining}

The total raw score on the entire CIS was used to determine level of goal-directed imagining. Using the framework of Wilson \& Barber (1978), four groups were created: high (25-40), medium high (22-24), medium low (15-21) and low (0-14).

\section{Suggestions of Coolness}

An additional item was created for the purpose of analysis of decrease in blood flow in response to hypnotic suggestion. The item consisted of suggestions based on those used by Bishay \& Lee (1984). Appendix B 
contains the item in its entirety.

\section{Blood Flow}

Changes in blood flow were assessed by measuring changes in localized skin resistance. Skin resistance of the surface of the right hand was measured by a Gulton \#45 thermister placed on the right hand. The thermister connected to a Fluke D800 digital voltmeter. Kilo-ohm measurements were recorded at regular intervals of 10 seconds throughout the course of the tape recording. Prior to the playing of the tape recording, a baseline measurement was obtained by taking the arithmetic mean of eight consecutive 10-second samples of localized skin resistance.

\section{CONDITIONS}

\section{Experimental}

Half of the subjects listened to a tape recording consisting of the hypnotic induction from the Stanford Hypnotic Clinical Scale for Adults (SHCS) (Morgan \& Hilgard, 1978) followed by the entire CIS and the added

coolness item. The tape ended with the arousal from hypnosis contained in the SHCS. The arousal was excerpted from the Amnesia item of the SHCS.

Control

The other half of the subjects listened to the same tape recording as the experimental group but without the induction section. The induction section was replaced by a passage of relaxing music of the same time 
length ( 8 minutes, 20 seconds) as the induction. The relaxing music consisted of the first 8 minutes, 20 seconds of Side A from the recording "Misty Forest Morning" (see References).

\section{PROCEDURE}

Subjects were screened for evidence of a history of dissociative reaction or for current use of neuroleptics or antidepressants. Those subjects who met either criteria for exclusion from the experiment were administered a short mock-experimental task and treated as if they were part of the actual study. Appendix C contains the screening instrument.

Wilson \& Barber (1978) contains the CIS. Prior to listening to the tape recording, subjects were instructed to read the following:

We are interested in the power of the imagination and the extent to which people may be able to use their imagination as a tool in relaxation and health. We appreciate your willingness to help in this study and hope that you will find this experience to be relaxing and enjoyable. You will sit in this chair, with your eyes closed, and listen to a short recording that is designed to help you to use your imagination ... we encourage you to just relax and have fun using your imagination while you follow the exercises on the recording.

The subject was then seated in a comfortable chair and the thermister was taped to the top surface of the subject's right hand approximately one inch below the base of the index finger in a region below the knuckle.

Experimental condition subjects were told:

In about a minute I'll start the tape. It starts out with around five minutes of progressive relaxation exercises. Then you'll go through a series of exercises so just follow along with the instructions 
In about a minute I'll start the tape. It starts out with around five minutes of relaxing music. Then you'll go through a series of exercises so just follow along with the instructions.

Subjects were run individually. Paired assignment to condition was determined on the basis of a coin toss. The total running time for the tape was 44 minutes. Once the tape had finished, the subject responded to the 10 CIS questions (see Appendix B) plus one added question asking for a scaled response, similar to those for the CIS items, to the added coolness item. The experimenter then debriefed the subject, answered any questions and thanked the subject for participation. 


\section{RESULTS}

\section{ANALYSIS OF OVERALL MODEL}

In order to test the effects of suggestion on blood flow, a repeatedmeasures analysis of variance (ANOVA) was performed. Kilo-ohm measurements were transformed into degrees Fahrenheit (F) through the application of the function:

$$
\mathrm{y}=-.675 \mathrm{x}+136.7
$$

where $y=$ degrees Fahrenheit and $x=$ kilo-ohms. A mean value of the dependent variable, skin temperature (ST), was associated with each of 14 levels of the treatment factor, time. These levels represented measurements taken during baseline, induction/music, CIS items 1-10, the added coolness item and the arousal interval. In addition to time, condition (experimental, control) and CIS group (high, medium high, medium low, low) were treated as factors in the analysis. Thus, the overall model consisted of a 2 X 4 X 14 design. Table I shows the mean ST for each treatment level by condition and for the overall analysis and Table II shows the various group sizes. The analysis revealed no main or interactive effects for condition or CIS group. A significant main effect for time, $F(13,364)=4.36, p<.0001$, did emerge, however. Figure 1 shows the time curves for the overall sample and for the two conditions. The curve for the overall sample was characterized by a sharp ST increase during the induction/music interval 
TABLE I

MEAN SKIN TEMPERATURE BY TREATMENT

Overall

Treatment Item

Baseline

Induction

Item 1

Item 2

Item 3

Item 4

Item 5

Item 6

Item 7

Item 8

Item 9

Item 10

Item 11

Arousal
$\mathrm{M} \quad \mathrm{SD}$

$85.8 \quad 4.06$

$86.8 \quad 4.02$

$87.4 \quad 3.94$

87.7

87.9

88.0

88.0

88.1

88.1

88.1

88.1

88.0

87.7

87.8
4.12

4.23

4.29

4.34

4.37

4.33

4.33

4.27

4.21

4.36

4.06
Experimental

$\mathrm{M} \quad \underline{\mathrm{SD}}$

$86.4 \quad 4.42$

$87.5 \quad 4.29$

$88.0 \quad 3.78$

$88.6 \quad 3.75$

$88.9 \quad 3.84$

$89.0 \quad 4.03$

88.9

89.0

89.1

89.1

89.0

88.9

88.4

88.8
Control

$\mathrm{M} \quad \underline{\mathrm{SD}}$

$85.3 \quad 3.71$

$86.2 \quad 3.76$

$86.8 \quad 4.10$

$86.9 \quad 4.41$

$87.0 \quad 4.50$

$87.1 \quad 4.45$

$87.2 \quad 4.42$

$87.2 \quad 4.37$

$87.1 \quad 4.29$

$87.1 \quad 4.31$

$87.2 \quad 4.22$

$87.1 \quad 4.11$

$86.9 \quad 4.04$

$86.7 \quad 3.88$

Note. All values represent degrees $F$

$\mathrm{M}=$ mean $\mathrm{SD}=$ standard deviation

TABLE II

GROUP SIZES: CONDITION AND CIS

$\underline{\text { Condition }}$

\section{CIS Group}

High

Medium High

Medium Low

Low
Experimental

5

4

5

4 $\underline{\text { Control }}$

3

5

4

6 


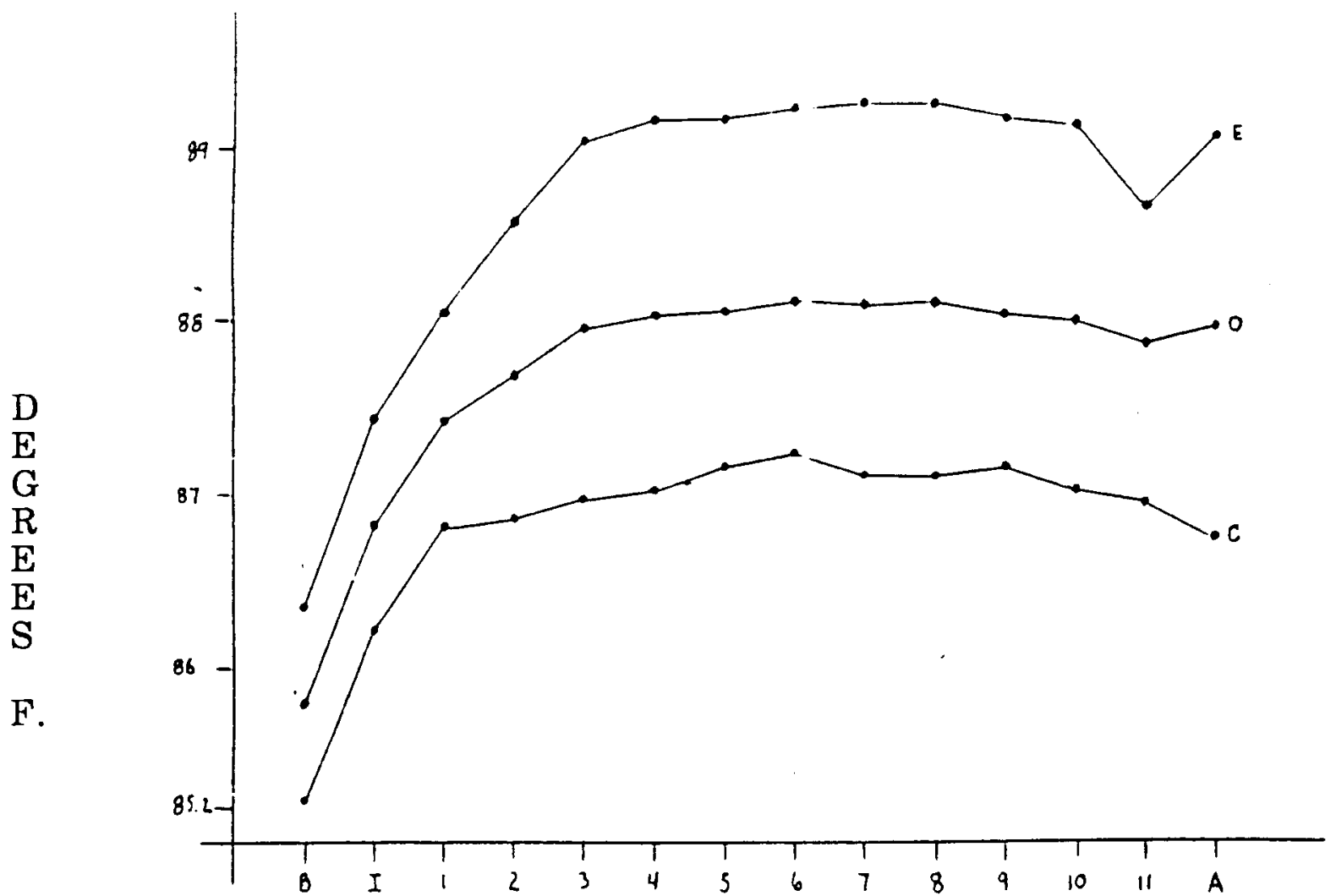

\section{TREATMENT ITEM}

Figure 1. Mean skin temperature as a function of treatment time. Note: $\mathrm{B}=$ baseline $\mathrm{I}=$ induction $\mathrm{A}=$ arousal $\mathrm{E}=$ experimental condition $\mathrm{C}=$ control condition $\mathrm{O}=$ overall sample 
and a gradual leveling-out around item 3 . This level trend continued until the coolness item (item 11) which was marked by a slight ST decrease of approximately .3 degrees $\mathrm{F}$, followed by a slight upward bend during the arousal interval. Orthogonal polynomial contrasts were generated to test the function of time for the overall sample. The contrasts resulted in significant main effects for the quadratic, $F(1,364)=8.10, p<.01$, and for the cubic, $F(1,364)=5.30, p<.05$.

\section{TEST OF HYPOTHESES}

Two 2 X 4 ANOVAs, using condition (2 levels) and CIS group (4 levels), were performed to test the differences between conditions on the items designed to increase (item 7) and decrease (item 11) blood flow to the right hand. Because the observed ST increase in item 1 may have been a continuation of the sharp trend initiated during the induction/music interval, the mean ST of items 2 through 6 was used as a measure against which the item $7 \mathrm{ST}$ was compared. Item 11 was compared with item 10. These two comparisons yielded two separate dependent variables (as defined by the differences) used in the ANOVAs. Separate ANOVAs resulted in no main effect for conditions on item $7, F(1,28)=.41$, not sig., nor on item $11, F(1,28)=2.20$, not sig.

To test the hypothesis of no difference between conditions on subjects' experiences of the suggested events, a two-way ANOVA employing condition and sex as predictors of total CIS score was performed. The range of CIS scores was 5-35 with a mean $(M)=20.11$ and a standard 
deviation (SD) of 7.54. No main or interactive effects emerged for either factor (including condition: $F(1,32)=1.07$, not sig.) although there was a tendency for the experimental group to score higher on the CIS ( $\mathrm{M}=21.44$, $\mathrm{SD}=7.54)$ than the control group $(\mathrm{M}=18.78, \mathrm{SD}=7.82)$.

To test the hypothesis that high CIS scorers would perform better than low scorers on the item designed to suggest decreases in blood flow, a paired t-test was performed. No difference emerged between high scorers $(n=8)$ and low scorers $(\mathrm{n}=10), \mathrm{t}=.467$, not sig., although both groups showed a slight tendency to decrease ST on this item. No test of the hypothesis that high CIS scorers would perform better than low scorers on the item designed to suggest increases in blood flow was performed due to the observation that high scorers actually exhibited ST decreases on the item.

To test the hypothesis that a rise in blood flow would occur in response to induction/music, a repeated-measures ANOVA was performed with two levels of time (baseline and induction). A significant main effect for time emerged, $F(13,28)=9.20, p<.001$. No main or interactive effects emerged for condition thus confirming the hypothesis of no difference between conditions on the expected ST rise from baseline through induction.

To test the hypothesis that high CIS scorers would demonstrate a greater baseline to induction rise in blood flow than low scorers, a t-test for paired differences was performed between the mean ST increases for each group. No difference between groups emerged, $t=1.30$, not sig., although the difference was in the expected direction. Table III summarizes the ST changes for the various groups. 
TABLE III

\section{MEAN SKIN TEMPERATURE CHANGES BY GROUP FOR INDUCTION/MUSIC, ITEM 7 \& ITEM 11}

Group

Overall

Experimental

Control

High CIS

Low CIS
Induction/Music

vs. Baseline

M SD

$1.03 \quad 1.77$

$1.11 \quad 1.58$

$.99 \quad 1.99$

2.13

.88
Item 7 vs. Mean of Items 2-6

$\underline{\mathrm{SD}}$

$.136 \quad .803$

$.224 \quad .836$

$.051 \quad .784$

$-.256 \quad 1.00$

$.144 \quad .843$
Item 11

vs. Item 10

M SD

$-.308 \quad 1.37$

$-.503 \quad 1.92$

$-.113 \quad .299$

$-.178 \quad .311$

$-.227 \quad .261$

Note. All values represent degrees $F$

$\mathrm{M}=$ mean $\mathrm{SD}=$ standard deviation 


\section{DISCUSSION}

The present study did not support the proposition that hypnotic suggestion can have an effect on the regulation of blood flow. It appeared, however, that administration of an induction resulted in a physiological response characterized by a significant increase in blood flow to the extremity. Since there was what could be interpreted as a "prolonged effect" of induction on blood flow through the first item, it could be that previous studies which claimed an effect for suggestion were measuring the effect of induction and not suggestion.

A claimed effect for hypnotic induction (specifically) does not seem warranted, however, due to the lack of difference between conditions on increased blood flow. Listening to relaxing music resulted in the same relative increase as receiving a standard hypnotic induction. If there is an effect on blood flow (and the data did, in fact, support this hypothesis) then the findings indicate that it should be attributed to relaxation and not to induction suggestions.

The failure to find an effect for suggestions of coolness raises an interesting possibility. Bishay \& Lee (1984) noted that, in some subjects, an increasing trend in blood flow tended to level off in response to suggestions of coolness. This aspect of their findings was actually supported by the present study in the sense that, following the effects of induction/music,the 
curve tended to flatten out. Hence, there is no reason to assume that small decreases in blood flow are the result of suggestions of coolness, per se.

Rather, they might be accounted for by the observation that, over time, the effect of induction lessens and, as a result, blood flow decreases.

Alternatively, it could be that a larger sample may yield results similar to those of Bishay \& Lee.

As predicted, the control group didn't differ from the experimental group on total CIS score. This finding supports a trait explanation in which experience of hypnotic phenomena is due, in part, to imaginative abilities. Whether or not entry into a special state is required for the intensive, optimal use of such abilities remains to be seen. The fact that the experimental group tended to score a few points higher suggests that there may be an ability by trance interactive factor involved in the experience of hypnotic events.

Although not a significant difference, subjects who scored high on the CIS demonstrated greater average increases in blood flow during the induction/music interval than did low scorers (in the expected direction). This suggests that goal-directed imagining may play a role in the experience of effective relaxation.

An anomaly which arose in the present study was the observation that low scorers on the CIS outperformed high scorers in the ability to decrease blood flow. While both groups demonstrated a mean decrease on the coolness item, the magnitude of the decrease was greater for the low scorers. Perhaps, since there was no overall effect for the coolness item 
the unexpected difference between these two groups should be downplayed. A better explanation, however, is that the hypnotic control of blood flow is simply not an observable phenomenon (in the sense that it doesn't occur) in controlled experimental conditions. Hence, the difference between these two groups would simply be due to small random deviations from the overall sample mean and not to a difference in goal-directed imagining.

Whether or not the hypnotic control of blood flow is an observable phenomenon in clinical settings remains an issue. Admittedly, tightlycontrolled experimental conditions characteristic of the present study may not adequately address the purported effects of hypnosis presented by researchers in clinical settings (e.g., Moore \& Kaplan, 1983; Erickson, 1980; Clawson \& Swade, 1975). It could be that the novelty of the experimental situation inhibited some subjects' willingness to imagine the suggested events. In support of this supposition, it was observed that the range of scores on the CIS was smaller than that reported by Wilson \& Barber (1978). Subjects in clinical settings may have habituated to certain characteristics of the hypnotist-client relationship which subjects in the present study remained vigilant toward.

It could be that an experimental design that incorporated use of a prolonged contact between subject and experimenter would, possibly, yield different results as may the training of select subjects over a prolonged period of time. In any event, future researchers in applied clinical settings should attempt to collect data on the relationship between suggestion and blood flow using physiological sampling techniques and standardized 
measures similar to those employed in the present study. Similarly, future researchers in experimental settings should employ longer sessions (perhaps, even longitudinal designs) and make specific attempts to introduce factors (e.g., rapport) that are known to affect hypnotic performance. 


\section{REFERENCES}

Barber, T. X. (1969). Hypnosis: A scientific approach. NY: Reinhold.

Barber, T. X. (1979). Suggested "hypnotic" behavior: The trance paradigm versus an alternative paradigm. In E. Fromm \& R. E. Shor (Eds.), Hypnosis: Developments in research and new perspectives, 2nd. edition (pp. 217-271). NY: Aldine.

Barber, T. X., \& De Moor, W. (1972). A theory of hypnotic induction procedures. American Journal of Clinical Hypnosis, 15, 112-135.

Barber, T. X., Spanos, N. P., \& Chaves, J. F. (1974). Hypnosis, imagination and human potentialities. NY: Pergamon.

Barber, T. X., \& Wilson, S. C. (1978). The Barber Suggestibility Scale and the Creative Imagination Scale: Experimental and clinical applications. American Journal of Clinical Hypnosis, 21, 84-108.

Bishay, E. G., \& Lee, C. (1984). Studies of the effects of hypnoanesthesia on regional blood flow by transcutaneous oxygen monitoring. American Journal of Clinical Hypnosis, 27, 64-69.

Clawson, T. A., \& Swade, R. H. (1975). The hypnotic control of blood flow and pain: The cure of warts and the potential for the use of hypnosis in the treatment of cancer. American Journal of Clinical Hypnosis, 17, 160-169.

De Horne, D. J., \& Powlett, V. (1980). Hypnotizability and rating scales. In G. D. Burrows \& L. Dennerstein (Eds.), Handbook of hypnosis and psychosomatic medicine (pp. 119-131). NY: Elsevier/North Holland.

Erickson, M. H. (1980). The hypnotic alteration of blood flow: An experiment comparing waking and hypnotic responsiveness. In E. L. Rossi (Ed.), Hypnotic alteration of sensory, perceptual, and psychophysicai processes (pp. 192-195). NY: Irvington.

Gordon, S. (1983). Misty forest morning environmental music. Distributed by Earth Light Center, 13906 Ventura Blvd., Sherman Oaks, CA 91423.

Grabowska, M. J. (1971). The effect of hypnosis and hypnotic suggestion on the blood flow in the extremities. Polish Medical Journal, 10, 1044-1051. 
Hammond, D. C., Keye, W. E., \& Grant, C. W. (1983). Hypnotic analgesia with burns: An initial study. American Journal of Clinical Hypnosis, 26, 56-59.

McConkey, K. M., Sheehan, P. W., \& White, K. D. (1979). Comparison of the Creative Imagination Scale and the Harvard Group Scale of Hypnotic Susceptibility, Form A. International Journal of Clinical and Experimental Hypnosis, 27, 265-277.

Moore, L. E., \& Kaplan, J. Z. (1983). Hypnotically accelerated burn wound healing. American Journal of Clinical Hypnosis, 26, 16-19.

Morgan, A. H., \& Hilgard, J. R. (1978). The Stanford Hypnotic Clinical Scale for Adults. American Journal of Clinical Hypnosis, 21, 134-146.

Sheehan, P. W., \& Perry, C. W. (1980). Research strategies in hypnosis: An overview of current methods. In G. D. Burrows \& L. Dennerstein (Eds.), Handbook of hypnosis and psychosomatic medicine (pp. 517-542). NY: Elsevier/North Holland.

Spanos, N. P., \& Barber, T. X. (1972). Cognitive activity during "hypnotic" suggestibility: Goal-directed fantasy and the experience of non-volition. Journal of Personality, 40, 510-524.

Spanos, N. P., \& Barber, T. X. (1974). Toward a convergence in hypnosis research. American Psychologist, 29, 500-511.

Spanos, N. P., \& Ham, M. W. (1973). Cognitive activity in response to hypnotic suggestions: Goal-directed fantasy and selective amnesia. American Journal of Clinical Hypnosis, 15, 191-198.

Spanos, N. P., McNeil, C., \& Stam, H. J. (1982). Hypnotically "reliving" a prior burn: Effects on blister formation and localized skin temperature. Journal of Abnormal Psychology, 91, 303-305.

Swirsky-Sacchetti, T., \& Margolis, C. G. (1986). The effects of a comprehensive self-hypnosis training program on the use of factor VIII on severe hemophilia. International Journal of Clinical and Experimental Hypnosis, 34, 71-83.

Wilson, S. C., \& Barber, T. X. (1978). The Creative Imagination Scale as a measure of hypnotic responsiveness: Applications to experimental and clinical hypnosis. American Journal of Clinical Hypnosis, 20, 235-243. 


\section{APPENDIX A}

\section{SUMMARY OF CIS ITEMS}

Item

1. Arm Heaviness

2. Hand Levitation

3. Finger Anesthesia

4. Water Hallucination

5. Gustatory Hallucination

6. Music Hallucination

7. Temperature Halluc.

8. Time Distortion

9. Age Regression

10. Mind-Body Relax

\section{Imagery of Suggestions}

One, two, then three heavy dictionaries being piled on top of the left hand

A strong stream of water from a garden hose is pushing up against the palm of the left hand

Novocain is being injected into the little finger of the left hand

Drinking water from a cool mountain stream

Eating a juicy, delicious orange

Listening to wonderful music heard in the past

The hot sun shining on the right hand

Time is slowing down

Feeling oneself back in elementary school

Lying on a beach, relaxed and at peace 


\section{APPENDIX B}

\section{COOLNESS ITEM}

Keeping your eyes closed, place your hands in your lap with the palms facing down and resting comfortably in your lap. By focusing your thinking, you can make your right hand feel cold.

Your right hand is feeling as if it is being pulled up ... as if there was a helium balloon attached to it with a strap and pulling it, very slowly, and slightly up. As your right hand leaves your lap, it is not going to go any higher. It feels cold and numb. You are immersing your right hand in a bucket full of ice-cold water. Your right hand is getting colder and colder, more and more numb ... your right hand is very cold and numb ... very cold, very numb. Move a finger on your right hand and feel it ... very cold. It is already pale and the veins are very collapsed. It feels cold and numb. You are immersing your right hand in a bucket full of ice-cold water. Your right hand is getting colder and colder, more and more numb ... your right hand is very cold and very numb ... cold, very cold, very numb.

Now tell yourself its all in your own mind and make your hand feel perfectly normal again ... make your hand feel perfectly normal again. 


\section{APPENDIX C}

\section{HEALTH STATUS QUESTIONNAIRE}

I understand that all information revealed in this questionnaire will be kept strictly confidential.

1. Have you ever had any of the following medical conditions?

__ Epilepsy

__ Fainting Spells

Rheumatic Fever

Frequent Loss of Balance

2. Have you ever suffered a severe head injury resulting in loss of consciousness, skull fracture or brain damage?

YES NO

3. Have you ever had a disturbed reaction to a hallucinogenic drug ?

YES NO

4. Have you ever had an unpleasant experience with chemical anesthesia?

YES NO

5. Do you have a history of sleepwalking ? If YES, how many years ago ?

YES NO

6. Are you currently taking an antipsychotic or antidepressant medication ?

YES NO 